\title{
Do the posterior midline cortices belong to the electrophysiological default-mode network?
}

\author{
Martin Sjøgård ${ }^{\mathrm{a}}$, Xavier De Tiège ${ }^{\mathrm{a}, \mathrm{b}}$, Alison Mary ${ }^{\mathrm{c}}$, Philippe Peigneux ${ }^{\mathrm{d}}$, Serge Goldman ${ }^{\mathrm{a}, \mathrm{b}}$, Guy Nagels ${ }^{\mathrm{e}, \mathrm{f}}$, Jeroen van \\ Schependom ${ }^{\mathrm{e}, \mathrm{g}}$, Andrew J. Quinn ${ }^{\mathrm{h}}$, Mark Woolrich ${ }^{\mathrm{h}}$, Vincent Wens ${ }^{\mathrm{a}, \mathrm{b}}$ \\ ${ }^{a}$ LCFC - Laboratoire de Cartographie fonctionnelle du Cerveau, UNI - ULB Neuroscience Institute, Université libre de Bruxelles (ULB), Brussels, Belgium \\ ${ }^{b}$ Magnetoencephalography Unit, Department of Functional Neuroimaging, Service of Nuclear Medicine, CUB - Hôpital Erasme, Brussels, Belgium \\ ${ }^{c}$ UNICAEN, PSL Research University, EPHE, INSERM, U1077, CHU de Caen, Neuropsychologie et Imagerie de la Mémoire Humaine, Caen, France \\ ${ }^{d} U R 2 N F$ - Neuropsychology and Functional Neuroimaging Research Unit at CRCN - Centre de Recherches Cognition et Neurosciences, and UNI - ULB \\ Neuroscience Institute, Université libre de Bruxelles (ULB), Brussels, Belgium \\ ${ }^{e}$ Center for Neurosciences, Vrije Universiteit Brussel, Brussels, Belgium \\ ${ }^{f}$ National MS Center Melsbroek, Melsbroek, Belgium \\ ${ }^{g}$ Radiology, UZ Brussel, Laarbeeklaan 103, Brussels, Belgium \\ ${ }^{h}$ Oxford Centre for Human Brain Activity (OHBA), University of Oxford, Oxford, UK
}

\begin{abstract}
The default-mode network (DMN) and its principal core hubs in the posterior midline cortices (PMC), i.e., the precuneus and the posterior cingulate cortex, play a critical role in the human brain structural and functional architecture. Because of their centrality, they are affected by a wide spectrum of brain disorders, e.g., Alzheimer's disease. Non-invasive electrophysiological techniques such as magnetoencephalography (MEG) are crucial to the investigation of the neurophysiology of the DMN and its alteration by brain disorders. However, MEG studies relying on band-limited power envelope correlation diverge in their ability to identify the PMC as a part of the DMN in healthy subjects at rest. Since these works were based on different MEG recording systems and different source reconstruction pipelines, we compared DMN functional connectivity estimated with two distinct MEG systems (Elekta, now MEGIN, and CTF) and two widely used reconstruction algorithms (Minimum Norm Estimation and linearly constrained minimum variance Beamformer). Our results identified the reconstruction method as the critical factor influencing PMC functional connectivity, which was significantly dampened by the Beamformer. On this basis, we recommend that future electrophysiological studies on the DMN should rely on Minimum Norm Estimation (or close variants) rather than on the classical Beamformer. Crucially, based on analytic knowledge about these two reconstruction algorithms, we demonstrated with simulations that this empirical observation could be explained by the existence of a spontaneous linear, approximately zero-lag synchronization structure between areas of the DMN or among multiple sources within the PMC. This finding highlights a novel property of the neural dynamics and functional architecture of a core human brain network at rest.
\end{abstract}

Keywords:

Functional connectivity; Linear synchronization; Magnetoencephalography; Resting state; Source reconstruction

Highlights:

- We investigate sources of inconsistencies in MEG DMN functional connectivity.

- MNE captures functional connectivity at the posterior midline cortices.

- LCMV Beamformer suppresses functional connectivity at the posterior midline cortices.

- We recommend using MNE instead of LCMV Beamformer for MEG studies of the DMN.

- Simulations suggest a spontaneous linear correlation structure within the DMN.

\section{Introduction}

A major discovery of the last couple of decades in human neuroscience has been that human brain activity appears intrinsically organized into large-scale functional networks known as resting-state networks (RSNs), each consisting of a set of remote brain areas preferentially coupled together (for a review,

${ }^{*}$ Corresponding author. Address: Laboratoire de Cartographie fonctionnelle du Cerveau, UNI - ULB Neuroscience Institute, Université libre de Bruxelles (ULB) and CUB - Hôpital Erasme, 808 route de Lennik, 1070 Brussels, Belgium. E-mail address: martin.sjogard@ulb.ac.be. see Deco and Corbetta, 2011). While most RSNs bind segregated regions involving neural activations during similar stimulation or task performance, one of them, i.e., the default-mode network (DMN), stands out. Indeed, its constituent regionsi.e., the posterior midline cortices (PMC) and more particularly the posterior cingulate cortex and the precuneus, the medial prefrontal cortex (mPFC), and the temporo-parietal junctions (TPJ) —were originally identified as those being metabolically less active during goal-directed tasks than at rest (Greicius et al., 2003; Raichle et al., 2001). This seminal discovery paved the way for fruitful research on the structure, dynamics 
and functions of the DMN, with promising critical implications for both fundamental neuroscience and clinical applications of functional neuroimaging (Fox, 2010; Fox and Raichle, 2007; Raichle, 2015). Structurally, the main regions of the DMN, especially those in the PMC, are among the most anatomically connected hubs of the brain (Hagmann et al., 2008; Sporns et al., 2007). Dynamically, the DMN is singled out as the most temporally unstable RSN (Allen et al., 2014). Furthermore, the PMC appears as one of the core hub systems enabling dynamic functional integration among the different RSNs (de Pasquale et al., 2012, 2016; for a review, see de Pasquale et al., 2018). Functionally, the DMN is involved in major human brain functions such as mind wandering and related spontaneous cognition (Buckner et al., 2008), sensory perception (Boly et al., 2007), or conscious processes (Baars et al., 2003) as well as in meditation (Marzetti et al., 2014, Xu et al., 2014 ). This multifaceted centrality of the DMN and the PMC also explains their implication in the pathophysiology of several major neurological and psychiatric disorders (Fox, 2010; Leech and Sharp, 2014).

The DMN and other RSNs have been uncovered from neuroimaging data mainly as spatial patterns of resting-state functional connectivity (FC), most prominently using functional magnetic resonance imaging (fMRI) (Fox and Raichle, 2007). Non-invasive electrophysiological recordings combined with source reconstruction and band-limited power envelope correlation also allowed the detection of cortical RSNs similar to the fMRI RSNs, both from magnetoencephalography (MEG) (Brookes et al., 2011; de Pasquale et al., 2010; Hipp et al., 2012) and electroencephalography (EEG) (Liu et al., 2017; Siems et al., 2016). Despite its wide use, fMRI is inherently limited in its ability to explore network dynamics (as it involves multiple timescales and preferential brain rhythms (Siegel et al., 2012)) and to make inferences about neural activity in pathological conditions or even physiological aging, as the neurovascular coupling may be altered (D'Esposito et al., 2003; Liu, 2013). Therefore, proper investigation of RSNs, and particularly of the DMN, using noninvasive electrophysiological techniques, appears of utmost importance. In this respect, investigating the electrophysiological FC of the DMN is especially crucial for clinical research on neurodegenerative disorders where results might diverge substantially from structural and fMRI studies (Coquelet et al., 2017).

Critically, although the DMN was recognizably identified in several electrophysiological studies, the MEG RSN literature is fraught with one key inconsistency regarding the detection of FC with the PMC: some studies identified all main DMN nodes except the PMC (Brookes et al., 2011; Hipp et al., 2012) or a moderate contribution of the PMC (Vidaurre et al., 2018), whereas others reported substantial FC with the PMC (de Pasquale et al., 2012; Wens et al., 2014). Clearly, this inconsistency has a major potential impact on subsequent inferences to be made about the neurophysiology of the DMN and its alteration by brain disorders.

To the best of our knowledge, this conundrum has not been explored yet, and here we fill that gap by investigating the possible factors at the source of these discordant findings. The main distinction between these previous works lies in the use of different types of MEG sensors and of different methods to reconstruct the neural sources underlying the measurements. Indeed, the studies reporting no contribution of the PMC in the DMN employed MEG systems composed of axial gradiometers together with a linearly constrained minimum variance (LCMV) Beamformer for source projection, whereas those reporting a strong contribution of the PMC in the DMN used systems involving magnetometers or planar gradiometers together with Minimum Norm Estimation (MNE) or close variants. Given the distinct sensitivity profiles of those sensors and the different assumptions behind those source reconstruction algorithms, we surmised that one, or a combination, of these two factors might explain the discrepancy in MEG DMN topographies. We investigated this empirically using human resting-state MEG data acquired with two different MEG systems and using two source reconstruction methods. We then considered network simulations to bring novel insights into the neurophysiological mechanisms at play in DMN functional dynamics.

\section{Materials and methods}

\subsection{Data collection and preprocessing}

We analyzed resting-state neuromagnetic recordings ( $5 \mathrm{~min}$, upright sitting, eyes open) obtained in two age- and sexmatched groups of fifteen right-handed healthy adults. One dataset ( 8 females; mean age: 24 years, age range: 19-30 years) was previously described and used (Wens et al., 2014). This data was acquired with an Elekta Neuromag system comprising 102 magnetometers and 102 pairs of orthogonal planar gradiometers, placed in a light-weight magnetically shielded room (Vectorview \& Maxshield ${ }^{\mathrm{TM}}$, Elekta Oy (now MEGIN, Croton Healthcare), Helsinki, Finland). Signal space separation (Taulu et al., 2005) was applied to suppress remnant environmental noise and correct for head movements, which were tracked using four head position indicator coils. The other dataset ( 8 females; mean age: 24 years, age range: $18-31$ years) was downloaded from the Open MEG Archive (Niso et al., 2016). This data was acquired on a CTF system comprising 275 first-order axial gradiometers with third-order synthetic gradient compensation and passive magnetic shielding for the suppression of environmental noise (VSM MedTech Inc., Coquitlam, BC, Canada). No head movement correction was applied to these CTF data but all subjects disclosed head displacement from the starting position to the end of the acquisition below $0.5 \mathrm{~cm}$.

Beyond the use of signal space separation with continuous movement correction for the Elekta MEG data, we applied the same preprocessing and analysis steps to the two datasets. Physiological noise was removed using independent component analysis (FastICA algorithm with dimension reduction to 30 and nonlinearity tanh (Hyvarinen and Oja, 2000)) of the band-pass filtered $(0.1-45 \mathrm{~Hz})$ data, visual detection and regression of the components corresponding to ocular and cardiac artifacts (Vigario et al., 2000). Cleaned data were then filtered in the alpha band $(8-12 \mathrm{~Hz})$, wherein the DMN was best identified in previous literature (Brookes et al., 2011; Wens et al., 
2014). Of notice, the Elekta data were further restricted to the 204 gradiometers for better comparability between the two systems. Each individual MEG recording was also accompanied with a high-resolution 3D T1-weighted cerebral magnetic resonance image (MRI) and a digitalization of fiducials, position indicator coils, and headshape points for manual coregistration of MEG and MRI coordinate frames.

\subsection{Source reconstruction algorithms}

A common source space was defined as a regular grid within the Montreal Neurological Institute (MNI) template brain volume and deformed onto each subject's MRI via a non-linear spatial normalisation (Ashburner and Friston, 1999) implemented in the SPM8 toolbox (Friston et al., 2007). The edge length of the MNI grid was set to $5 \mathrm{~mm}$, but given the potential impact of too coarse source sampling on the LCMV Beamformer (Van Veen, 1997) we also considered a finer grid with 2-mm long edges. Individual MEG forward models were computed using the single-layer boundary element method of MNEC (Gramfort et al., 2014), which is itself based on the MRI tissue segmentation performed with FreeSurfer (Fischl, 2012).

Two different source reconstruction algorithms were then applied on the rest data: (i) MNE (Dale and Sereno, 1993) with noise covariance estimated from $5 \mathrm{~min}$ of empty-room recordings (with environmental noise cancellation) filtered in the alpha band and regularization parameter set through a prior consistency condition (Wens et al., 2015), and (ii) a unit-gain LCMV Beamformer (Hillebrand et al., 2005; Van Veen, 1997) with covariance estimated from the alpha-band resting-state data. Of note, prior to Beamforming, each three-dimensional forward model dipole was projected onto the plane orthogonal to its direction of negligible magnetic response. Finally, in both cases, reconstructed source time series were projected onto their direction of maximum variance (Brookes et al., 2011; Wens et al., 2014).

\subsection{Electrophysiological functional connectivity}

Power envelope FC between two source signals was estimated as the Pearson correlation of their Hilbert envelopes lowpass filtered at $1 \mathrm{~Hz}$ using sliding-window averaging (window width: $1 \mathrm{~s}$, sliding step: $0.5 \mathrm{~s}$ ). Spatial leakage was corrected beforehand using pairwise static orthogonalization (Brookes et al., 2012) so as to eliminate completely any linear, zero-lag coupling and also minimize correction errors due to source mislocalization (Wens et al., 2015).

We estimated the DMN topography using seed-based FC mapping with a seed placed at the mPFC (MNI coordinates: $[-2,51,2] \mathrm{mm}$ ) as in a previous study (Wens et al., 2014) because this node is well isolated from the other DMN nodes, so this choice minimizes interpretation ambiguities related to local miscorrection of spatial leakage or ghost interactions potentially surviving after orthogonalization (Palva et al., 2018; Wens et al., 2015).

For a more quantitative, data-driven analysis, we then considered a functional connectome built by estimating FC among 38 non-overlapping regions of interest (ROIs) covering the whole brain. These ROIs were based on a functional brain parcellation derived from a high-dimensional group independent component analysis of resting-state fMRI data (Colclough et al., 2015). Each of the associated weighted ROI maps were binarized to build a mask identifying the nonzero weights, and the set of source signals within each ROI mask was reduced to one representative signal that maximally accounted for the variance within the ROI, i.e., its principal component. The resulting 38by-38 FC matrix was explicitly symmetrized to avoid potential asymmetries induced by pairwise orthogonalization (Brookes et al., 2012; Hipp et al., 2012). Global connectivity was assessed by averaging over all connections, and a matrix of regional FC was then obtained by subtracting this global value from each entry. A two-way mixed ANOVA was used to examine the possible effects of MEG system type (between-subject factor, two levels) and source reconstruction type (within-subject factor, two levels) on both global and regional FC. In the latter case, the family-wise error rate (FWER) was controlled by estimating the number $N_{\text {dof }}$ of degrees of freedom associated with the 38 reconstructed signals, which may be less than 38 due to spatial leakage effects among ROIs. To do so, we extracted the inverse operators associated with these signals (i.e., their projection onto the corresponding principal component) and computed their rank as described in Wens et al. (2015). These ranks varied a little across reconstruction methods and system types (Elekta-MNE: 22, Elekta-LCMV Beamformer: 25, CTF-MNE: 21, CTF-LCMV Beamformer: 23) so we used the maximum value, $N_{\text {dof }}=25$, leading to only slightly conservative testing. The Bonferroni correction factor applied to the connectomelevel ANOVA was thus $N_{\text {fwer }}=N_{\text {dof }}\left(N_{\text {dof }}-1\right) / 2$ and the corresponding significance level, $p<0.05 / N_{\text {fwer }}=1.7 \times 10^{-4}$.

\subsection{Simulations of the default-mode network}

Given the known inability of the LCMV Beamformer to reconstruct correlated sources (Hillebrand et al., 2005), we reasoned that any systematic differences between MNE- and LCMV Beamformer-based resting-state FC could be related to a spontaneous linear correlation structure within the DMN. Thus, we generated synthetic data simulating two different models of the DMN (using the forward models and measurement noise recordings from the two datasets) and then applied the exact same FC analysis pipeline as for the human restingstate data.

Each group-level simulation consisted in the generation of fifteen synthetic MEG data (one for each subject of a restingstate dataset) built from a simulated DMN configuration together with an additive brain noise background. The DMN sources were either placed at isolated nodes (mPFC: $[-2,51,2]$ mm, PMC: $[-3,54,31] \mathrm{mm}$, left TPJ: $[-43,-76,35] \mathrm{mm}$, right TPJ: [43, -76,35] mm; MNI coordinates (de Pasquale et al., 2010)) or covered corresponding ROIs. Each dipole orientation was fixed along the direction of maximum MEG response (as assessed by the individual forward model), and source time courses were obtained from independent realizations of an alpha-band filtered Gaussian white noise (sampling rate $f_{s}: 200$ $\mathrm{Hz}$, duration: $5 \mathrm{~min}$ ) or from linear combinations thereof to investigate the effect of linear, zero-lag coupling on FC mapping. 
Specifically, a linear PMC-TPJ coupling was introduced by replacing the PMC node signal with an interpolation between the original PMC signal (no linear correlation) and the sum of the left and right TPJ signals (maximum PMC-TPJ correlation: $1 / \sqrt{2} \approx .7$ ). Within-PMC synchronization was generated by interpolating between the case of linearly independent source signals in the corresponding ROI (no linear correlation) and the case of all identical signals in the ROI (maximum mean withinPMC correlation: 1). Importantly, these linear couplings were simulated as instantaneous interactions, but given our restriction to the alpha band they can also be interpreted as reflecting synchrony with small but non-zero time delays. To make this point explicit, we will refer to these as being "zero-lag". Nonlinear FC was then imposed among all the DMN sources by weighting their signals at each time point using a common .1 $\mathrm{Hz}$ sinusoid, which allows co-modulating the Hilbert envelopes while leaving the linear correlation structure relatively intact (Brookes et al., 2012). Of note, the variance of the resulting source time courses were normalized to one except in the case of PMC simulated as an isolated node, for which the effective decrease in gradiometer signal (which falls off according to the inverse cubed distance between source and sensors) due to its depth was counter-balanced by increasing its variance appropriately.

The background brain noise was generated for each source location using independent realizations of an auto-regressive model that roughly mimics the temporal autocorrelation structure of human MEG data, which is dominated by strong alpha power around $10 \mathrm{~Hz}$. Specifically, we chose a model order of two and fixed the roots of the characteristic polynomial to $z=0.8 \exp \left(2 \mathrm{i} \pi \times 10 \mathrm{~Hz} / f_{s}\right)$ and its complex conjugate. Brain noise variance was set to $10 \%$ of the DMN signal's variance. Finally, the synthetic MEG data were obtained by individual forward projection of the resulting source configuration and addition of measurement noise taken from empty-room MEG recordings. The latter were rescaled beforehand so as to fix the signal-to-noise ratio (SNR) of the synthetic MEG data to the value estimated from the corresponding subject's resting state. Source reconstruction and FC analysis of all simulated MEG data were performed as for the corresponding resting-state data. A two-way ANOVA with FWER correction was applied on the resulting regional $\mathrm{FC}$ estimates to assess the effects of source reconstruction type and of the presence/ absence of linear synchronization (within-subject factor, two levels).

\subsection{Data and code availability}

CTF data is available from the Open MEG Archive (Niso et al., 2016). Elekta data and code is available upon reasonable request to the authors.

\section{Results}

\subsection{Empirical analysis of DMN connectivity at rest}

We show in Figs. 1 and 2 the results from four group-level analyses of the DMN (15 subjects in each group), using alphaband power envelope FC with leakage correction estimated on

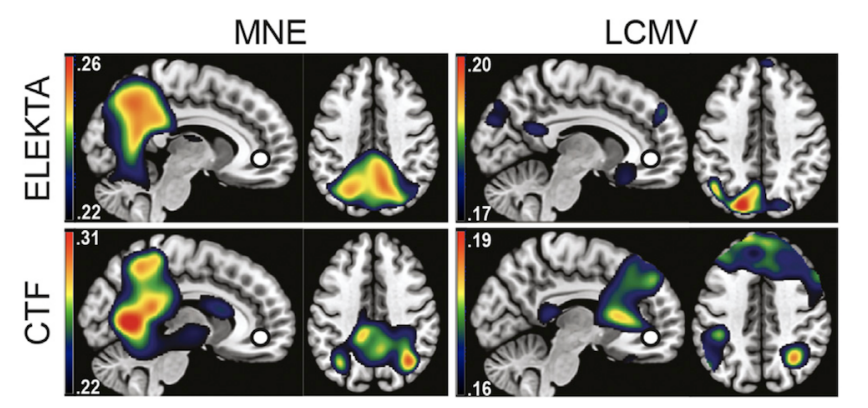

Figure 1: Empirical DMN connectivity at rest. Seed-based FC maps with a seed at the mPFC (white disc) for both reconstruction methods (MNE and LCMV Beam- former) and both system types (Elekta and CTF). For visualization purposes, topographical maps were thresholded so as to disclose the $20 \%$ strongest connections.

a 5-mm source grid. We considered two source reconstructions (MNE and LCMV Beamformer) applied to two restingstate datasets acquired with different MEG systems (Elekta and CTF). As seen in Fig. 1, the classical DMN couplings between the $\mathrm{mPFC}$ seed and the PMC and TPJs emerged using MNE, hence disclosing a full DMN topography as found in fMRI (Smith et al., 2009). By contrast, the mPFC-PMC coupling was notably absent in the LCMV Beamformer-based maps. This was true for both MEG systems, suggesting a difference dominated by the reconstruction algorithm. Frontal FC was better seen when using the LCMV Beamformer, especially in the CTF data; however this is difficult to interpret due to potential local miscorrection of spatial leakage (Wens et al., 2015).

To quantify these observations statistically and without priors about the DMN, we considered a functional connectome encompassing FC between 38 ROIs covering the whole brain (Fig. 2). Given the large differences in the scales of MNEand LCMV Beamformer-based FC matrices, we first applied an ANOVA on the global FC and identified a significant main effect of reconstruction $\left(F_{1,56}=37.2, p=1.1 \times 10^{-7}\right)$ but not of system type or any interaction effect $\left(F_{1,56} \leq .6, p \geq .44\right)$. Because this global effect does not affect the relative FC values among the connections, we then performed an ANOVA on the regional FC estimates, and the results disclosed a substantially significant main effect of reconstruction on the coupling between ROIs 21 and $22\left(F_{1,56}=27.4, p=2.6 \times 10^{-6}\right)$, which correspond respectively to the MPFC and PMC nodes (Fig. 2, bottom). Reconstruction effects did not reach significance for any other connection $\left(F_{1,56} \leq 7.9, p \geq 6.7 \times 10^{-3}\right)$. Importantly, we observed no main effect of system type $\left(F_{1,56} \leq 1.8\right.$, $p \geq .19)$ nor any interaction $\left(F_{1,56} \leq 2.4, p \geq .13\right)$.

These empirical results are robust in that they did not depend on the precise choice of the seed location (Supplementary Material S1, Fig. S1) or the shape of the ROIs (Supplementary Material S2). Importantly, FC analysis based on a finer 2-mm source space also showed that the LCMV Beamformer-specific absence of FC between the mPFC and the PMC was not merely related to our choice of the source grid (Supplementary Material S3, Fig. S2). The impact of the precise method to estimate envelope FC was also fairly limited (see Supplementary Mate- 
Elekta LCMV

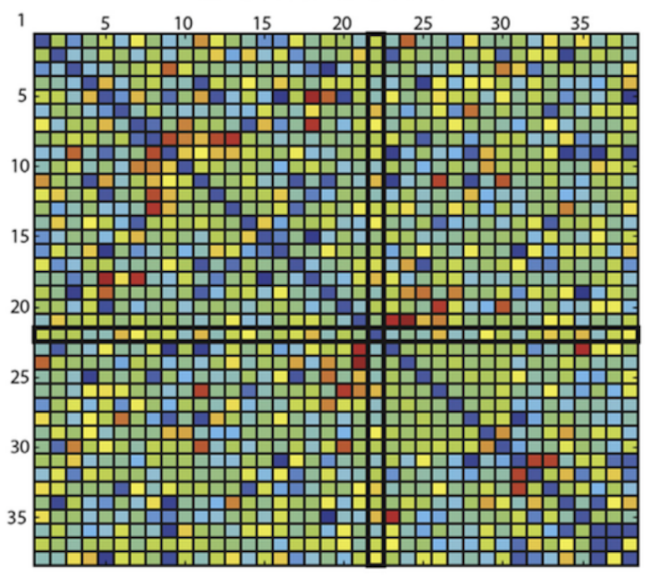

Elekta MNE

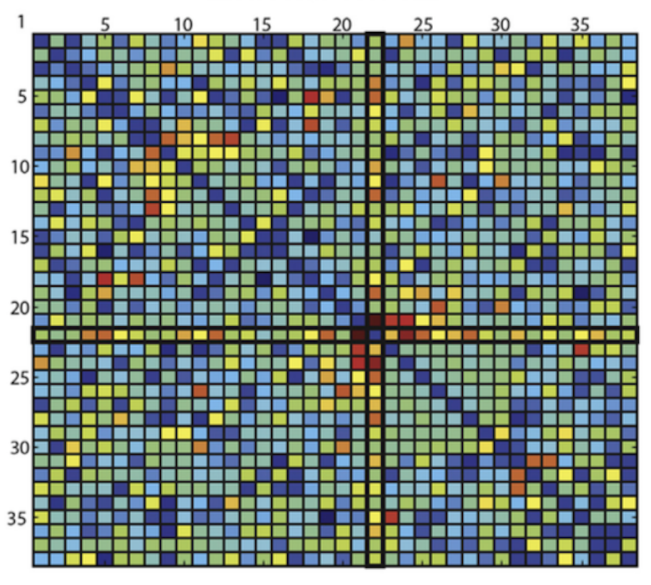

CTF LCMV

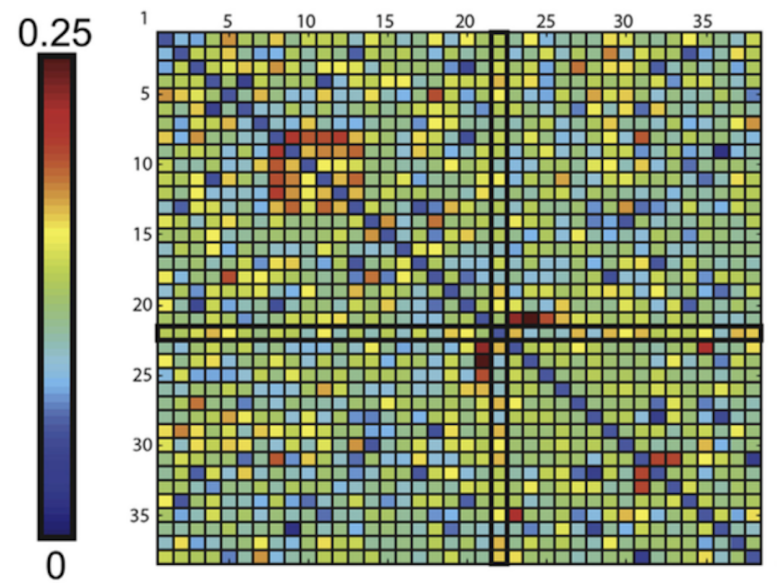

CTF MNE

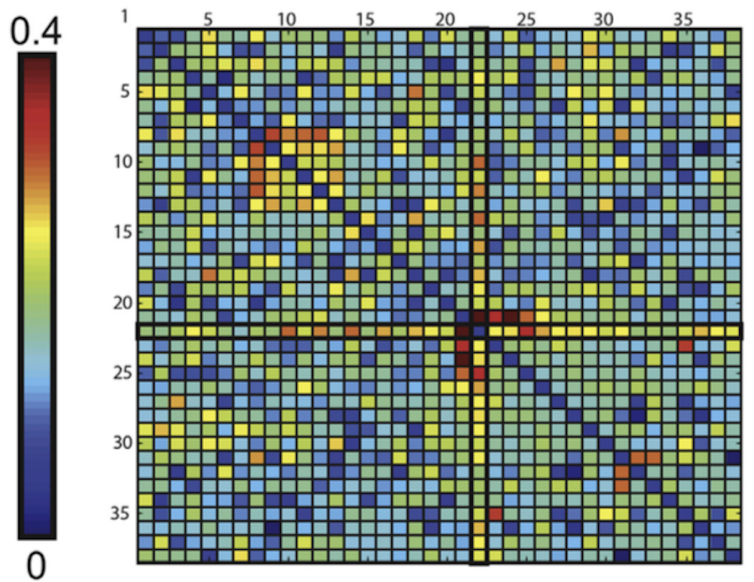

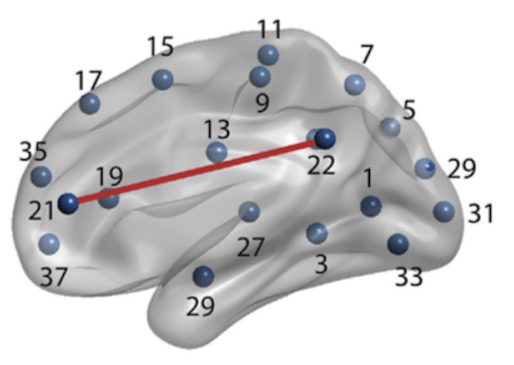
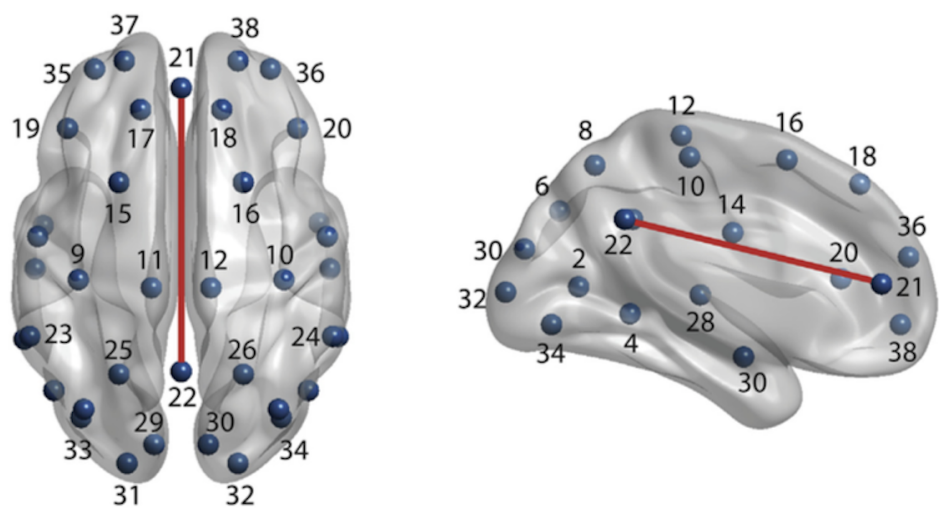

Figure 2: Empirical functional connectome at rest. The FC matrices are shown for the four combinations of MEG system (Elekta and CTF) and source reconstruction algorithm (LCMV Beamformer and MNE). The center of mass of the corresponding ROIs (blue nodes) as well as connections with a significant effect of reconstruction, system type, or their interaction (ANOVA, $p<0.05$ FWER corrected; red edges) are located on the MNI glass brain on the bottom. The FC matrices' rows and columns corresponding to the significant connections are also emphasized in bold. 
rial S4).

\subsection{Hypothesis-driven simulations of the DMN}

We further explored why the strong reconstruction effect takes place for the mPFC-PMC resting-state FC using two simulated models of the DMN (Figs. 3 and 4). Given the lack of system effect, we focused for definiteness on synthetic data generated from the Elekta dataset.

\subsubsection{Point-like PMC source with linear PMC-TPJ coupling}

In a first scenario we simulated the DMN as four wellseparated pointlike sources (mPFC, PMC, TPJs) that were envelope correlated but with no linear "zero-lag" connectivity, and analyzed the effect of introducing bilateral "zero-lag" correlations between the PMC node and the TPJ nodes (Fig. 3). The introduction of these correlations between the PMC and the TPJs reproduced quite clearly our observation that the mPFCPMC coupling could be detected using MNE but not LCMV Beamformer, while the mPFC-TPJ couplings were detectable with or without them (Fig. 3A, bottom row). This contrasts with a control simulation without any linear coupling between the PMC and the TPJs, where both reconstruction methods detected a full DMN (Fig. 3A, top row). These observations were confirmed by a quantitative ANOVA of the functional connectome (regional FC matrix) with reconstruction and linear dependence (i.e., zero or maximum PMC-TPJ correlation) as factors (Fig. 3B). The mPFC-PMC connection was singled out by a main effect of both reconstruction $\left(F_{1,56}=17.4, p=1.1 \times 10^{-4}\right)$ and linear dependence $\left(F_{1,56}=28.9, p=1.5 \times 10^{-6}\right)$ as well as their interaction $\left(F_{1,56}=43.2, p=1.8 \times 10^{-8}\right)$. No other significant effect was identified $\left(F_{1,56} \leq 7.5, p \geq 8.5 \times 10^{-3}\right)$.

We assessed the PMC-TPJ correlation strength needed to reproduce this LCMV Beamformer-specific loss of mPFC-PMC connectivity. Examples of LCMV Beamformer-based FC maps for high PMC-TPJ correlations illustrate a rapid disappearance of the PMC as this correlation approached its maximum value (Fig. 3C). To confirm this, we show in Fig. 3D the mPFC-PMC coupling estimate as a function of the PMC-TPJ correlation. The plot for MNE appeared constant, consistent with the absence of impact of linear PMC-TPJ connectivity. The plot for the LCMV Beamformer was more variant but still remained around a definite value over a quite large range of linear correlations (from 0 to about .6) and then dropped sharply and vanished at the maximum possible value (about .7).

Of note, in these point-source simulations, the signal variance at the PMC node needed to be increased by a factor related to the subject-specific ratios of PMC to TPJ distance to the MEG sensors, in order to compensate for its lower SNR due to its cortical depth. Indeed, similar simulations failed to disclose any PMC connectivity when the simulated PMC power was too low (Supplementary Material S5, Fig. S3). Similarly, other control simulations were performed to inspect the network independence of global FC decreases induced by the LCMV Beamformer (Supplementary Material S6, Fig. S4) and check the necessity of bi-laterality in the linear PMC-TPJ coupling model (Supplementary Materials S7, Fig. S5).

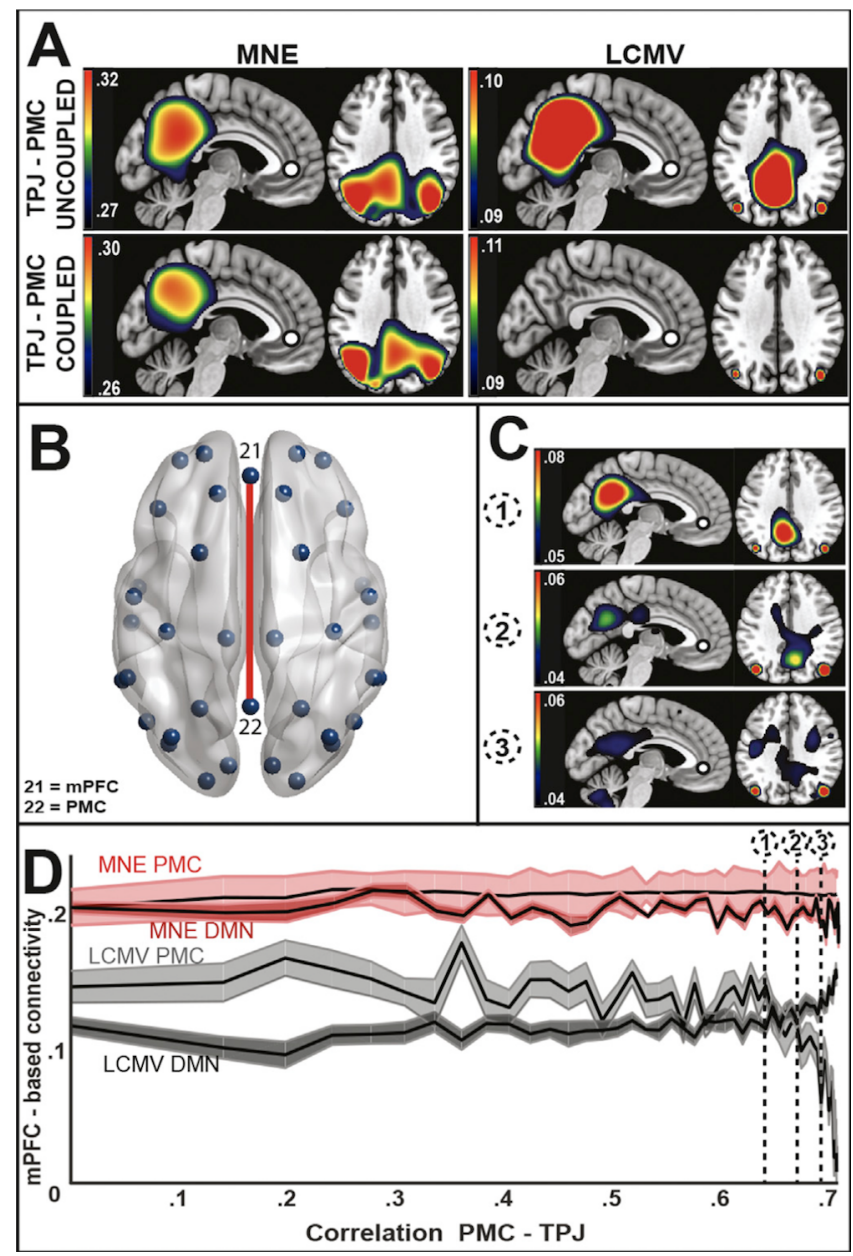

Figure 3: Effect of bilateral linear PMC-TPJ coupling on simulated DMN. (A) Resulting mPFC-based FC maps for both reconstruction methods (MNE and LCMV Beamformer) and both the presence (bottom row) or absence (top row) of linear correlations. (B) Connections disclosing a significant effect of reconstruction, linear dependence, or their interaction (ANOVA, $p<0.05$ FWER corrected). (C) Evolution of the mPFC-based FC using LCMV Beamformer for selected linear correlation values (emphasized by dotted lines in panel D). (D) Mean (black lines) and SEM (colored margins) of mPFC-based FC values averaged within the PMC parcel as a function of the linear PMC-TPJ correlation, using MNE (light red) and LCMV Beamformer (light grey). For comparison, mPFC-based DMN FC (i.e., averaged across the PMC and the left and right TPJ parcels) is also shown (MNE: dark red, LCMV Beamformer: dark grey). 


\subsubsection{Spatially extended linear synchronization within the $P M C$}

In a second scenario, we instead considered the effect of local synchronization within an extended PMC modeled by placing multiple point sources within the PMC region (Fig. 4). Comparing the situation where all within-PMC sources were identical (fully synchronized PMC) to the situation where all were linearly independent (asynchronous PMC) reproduced results similar to those of the point-like PMC simulation (Fig. 4A and B). The mPFC-PMC coupling was once again singled out by a main effect of reconstruction $\left(F_{1,56}=14.5, p=3.5 \times 10^{-4}\right)$ but not of PMC synchronization (i.e., mean within-PMC correlation: 0 or $\left.1 ; F_{1,56}=.92, p=.34\right)$ and, more importantly, there was a strong interaction $\left(F_{1,56}=36.1, p=1.5 \times 10^{-7}\right)$. No other effect was identified $\left(F_{1,56} \leq 9.0, p \geq 4.0 \times 10^{-3}\right)$. The behavior of the mPFC-PMC coupling estimate as we varied the strength of within-PMC synchronization is shown in Fig. 4C and D. Fig. 4D appeared qualitatively different from the analogous Fig. 3D in the first model. Indeed, the LCMV Beamformer-based FC decreased with a linear trend as PMC synchronization increased and crossed below the global withinDMN FC (which remained stable) at a mean within-PMC correlation of about .3. For MNE, both the MPFC-PMC and the global within-DMN FC increased but their difference was stable, so PMC synchronization had no effect on regional FC.

\section{Discussion}

This study demonstrated that electrophysiological mapping of the DMN via power envelope correlations based on the LCMV Beamformer is relatively blind to PMC connectivitymore precisely, to the antero-posterior coupling between the $\mathrm{mPFC}$ and the PMC - whereas those based on MNE disclose a prominent contribution. These observations were robustly reproducible across two different MEG systems, i.e., Elekta and CTF neuromagnetometers, which is in line with the MEG literature (Brookes et al., 2011; de Pasquale et al., 2010; Wens et al., 2014). They also did not depend on technical details such as, e.g., source space sampling. Crucially, network simulations showed that this discrepancy could originate from the existence of a spontaneous linear, "zero-lag" synchronization structure between areas of the DMN or among multiple sources within the PMC.

\subsection{The PMC is a part of the electrophysiological DMN}

The first question prompted by these data is whether the PMC belongs to the electrophysiological DMN. This hypothesis is strongly supported by our results and the existing literature, as the dominating PMC connectivity identified with MNE is similar to that observed in fMRI (Smith et al., 2009) and positron emission tomography (Savio et al., 2017; Trotta et al., 2018). Actually, assuming that the LCMV Beamformer provides an accurate picture of the DMN (i.e., without PMC) leads to the implausible conclusion that PMC connectivity is a purely vasculometabolic phenomenon that is nevertheless spuriously reconstructed by MNE. Furthermore, we provided simulation-based

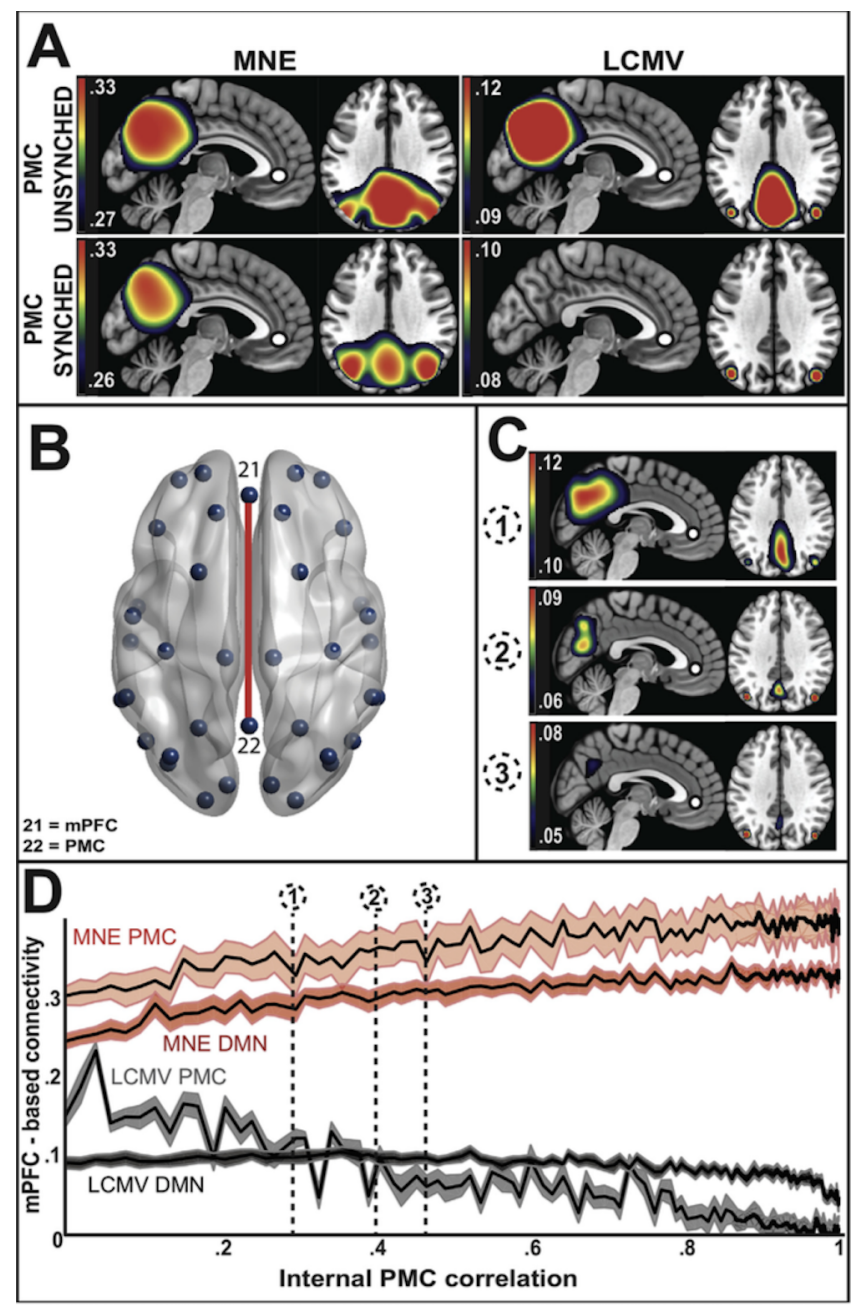

Figure 4: Effect of linear within-PMC synchronization on simulated DMN. All is as in Fig. 3. 
evidence that MNE does not project sensor signal at the PMC area in the absence of true PMC activity (Supplementary Material S5, Fig. S3).

Conversely, accepting the neural nature of the PMC connectivity implies that the LCMV Beamformer substantially underestimates some PMC-based interactions within the DMN. Actually, we identified both a global connectivity decrease and a local decrease of the mPFC-PMC coupling with this reconstruction method. The former corresponds to a general dampening of connectivity by the LCMV Beamformer that is irrespective of the underlying brain activity, as it was also observed in pure noise simulations (see Supplementary Material S6, Fig. S4). This is due to the cross-sensor correlations induced by the magnetic field spread, which dominate over the correlations generated by neural activity. This intrinsic covariance structure necessarily leads to global source dampening by the LCMV Beamformer. On the other hand, the localized effect could not be accounted for by this global decrease alone. Specifically, the mPFC-PMC coupling was reduced to the point of not being distinguishable from background connectivity, whereas the other mPFC-TPJ connections of the DMN did still emerge from the background. This suggests a reconstruction bias of restingstate activity related to the PMC when using the LCMV Beamformer methodology. This has both major conceptual and practical consequences.

\subsection{Linear synchronization structure at rest}

We sought to explain the PMC-specific differences between MNE- and LCMV Beamformer-based functional connectomes by assuming the existence of genuine linear correlations associated with PMC neural dynamics. Indeed, the LCMV Beamformer is known to suppress linearly correlated sources (Hillebrand et al., 2005). An alternative, more technical possibility would have been a sampling bias due to a too coarse source grid. Indeed, the LCMV Beamformer is unable to reconstruct a focal source located outside the grid in situations of too large SNR where the typical width of its point-spread functions is below the grid size (Van Veen, 1997). That said, resting-state activity presumably does not exhibit such SNR levels. Accordingly, using a finer grid did not allow recovery of the mPFCPMC coupling with the LCMV Beamformer (Supplementary Material S3, Fig. S2). On this basis, we thus examined two possible neurophysiological scenarios, based on either (i) a linear, "zero-lag" coupling between the PMC and other areas of the DMN, or (ii) a local linear, "zero-lag" synchronization structure within the PMC.

In the first scenario, we could exclude from scratch the possibility of linear, "zero-lag" coupling with the mPFC node because our seed-based maps were obtained after signal orthogonalization with that node, which by design eliminated not only spatial leakage effects but also any possible physiological linear, "zero-lag" correlations with the mPFC (Wens et al., 2015). The only remaining possibility was thus the existence of lateral linear correlations between the PMC and the TPJs, and because of the bi-laterality of the DMN a similar amount of linear mixing between the PMC and the left and right TPJs was expected (as checked in Supplementary Material S7, Fig. S5). Simulating such configuration indeed reproduced clearly the LCMV Beamformer-specific disappearance of the mPFC-PMC coupling and its successful identification with MNE, as well as the relative preservation of connectivity with the TPJs. The preservation of the TPJs is explained by the fact that a certain degree of independence between the two TPJ signals was allowed. By contrast, the PMC source was built deterministically from the two TPJ signals (at least for maximal PMC-TPJ correlation), which led to its cancellation by the LCMV Beamformer.

Importantly, this scenario focused on the coupling dynamics between nodes of the DMN, which were merely simulated as point sources, and thus mostly neglected possible effects of the internal structure within each constituent area of the DMN (except for the amplitude boost needed for the PMC node, see below). Alternatively, in the second scenario, we examined the effect of linear correlation within the PMC independent of any linear, "zero-lag" connectivity between DMN areas. Simulating PMC configurations using a spatially-extended distribution of synchronized sources also replicated clearly our empirical observations about the mPFC-PMC coupling. Here, the preservation of TPJ connectivity was built in, since the TPJ signals were simulated as independent sources.

These two scenarios provide distinct neurophysiological mechanisms that reproduced equally well the LCMV Beamformer-specific disappearance of the mPFC-PMC coupling, and this prompts the question of whether one is more likely than the other. Notwithstanding the fact that they are not exclusive, we argue here that the extended PMC synchronization model is more plausible than the linear PMC-TPJ coupling model. One key element that allows them to be distinguished comes from our simulations where the degree of synchrony was varied. As expected, both the LCMV Beamformer and MNE were able to disclose the PMC connectivity correctly in the absence of any linear correlation, and increasing linear correlations had little effect when using MNE. On the other hand, the LCMV Beamformer-based FC between the mPFC and the PMC decreased in different ways according to the scenario considered, and disappeared below the global within-DMN FC above a critical correlation value of about .3 for the PMC synchronization model and about .6 for the linear PMC-TPJ coupling model. In the latter case, the level of linear correlation needed to reproduce the empirical resting-state data was thus substantial and presumably beyond a reasonable physiological range. Actually, this large critical value corresponds to a situation where the PMC signal is an almost perfect mix of the two TPJ signals, which conflicts with the idea that the PMC encompasses a major integrative hub of the human brain (de Pasquale et al., 2012; Leech and Sharp, 2014). Another argument favoring the extended PMC synchronization model is the observation that the linear PMC-TPJ coupling model actually implicitly involved an extended PMC, despite the point-like nature of the simulations, as we had to boost the PMC node amplitude in order to adjust for its depth and reproduce the empirical data. From a neurophysiological perspective, increased local power amplitude is often assumed to reflect a larger synchronization area for post-synaptic potentials (Buzsaki et al., 2012). Ad- 
ditionally, the extended PMC scenario is compatible with the well-described structural and functional heterogeneity of the precuneus and the posterior cingulate cortex, which splits the PMC into at least two sub-areas (Cavanna and Trimble, 2006; Leech and Sharp, 2014; Margulies et al., 2009; Utevsky et al., 2014; Vogt et al., 2006). Taken together, these arguments thus favor the idea of a linear synchronization within the PMC. That said, no definite hard conclusion can be drawn since testing these linear synchronization models directly in MEG data is intrinsically limited by spatial leakage among close areas such as the PMC and the TPJs.

Whatever the precise scenario involved, our empirical and simulated data suggest the existence of genuine linear, "zerolag" couplings besides the nonlinear power correlations that are the electrophysiological signatures of RSNs. Interestingly, the opposite hypothesis-i.e., the absence of linear, zero-lag correlations-was explicitly used as a basic assumption for the development of RSN mapping based on the LCMV Beamformer (Brookes et al., 2011) and signal orthogonalization for spatial leakage correction (Brookes et al., 2012; Colclough et al., 2015). This led to a nicely internally consistent methodological approach that was also partly justified by O'Neill et al. (2015), who examined the similarity of LCMV Beamformerand MNE-based RSN maps, although, critically, the DMN was not included there. Our analysis of a functional connectome confirmed and extended this observation, i.e., both LCMV Beamformer- and MNE-based FC estimation within and across RSNs are similar except for within-DMN couplings. Crucially, this exception indicates that this working hypothesis about the resting state may not be generally valid, at least when it comes to the DMN. However, our data provide no indication as to why the putative spontaneous linear, "zero-lag" interactions are restricted to the DMN and what their function might be. One clue may be the observation, using intracerebral stereotactic EEG recordings, of event-related gamma-band desynchronization occurring within DMN areas (Jerbi et al., 2010). In view of its non-specificity to the precise goal-directed task considered and of the high frequencies involved (i.e., $50-150 \mathrm{~Hz}$ ), this result suggests that the DMN exhibits at rest a lagged correlation structure associated with time delays on the order of 5-20 ms. As already noted in the Methods section, since we analyzed the DMN using FC in the alpha band wherein such fast temporal fluctuations are inaccessible, this is compatible with our simulated zero time lags. On this basis, we can thus speculate that the putative spontaneous linear and "zero-lag" correlations of the DMN essentially reflect its inner-directed nature, and their absence in other RSNs, their outer goal-directed nature. This insight highlights a novel aspect of the functional dynamics of the electrophysiological DMN in the resting state.

\subsection{A practical guideline for DMN resting-state studies}

The successful identification of PMC connectivity by MNE and its loss with the LCMV Beamformer have a very practical implication for the methodological choices to make in studies dedicated to the electrophysiology of the DMN (and more particularly of the PMC). Namely, we recommend that such endeavors reconstruct brain FC based on MNE or close variants rather than on LCMV Beamformer. This is analogous to the guidance offered by Hincapié et al. on coherence-based FC analysis (Hincapié et al., 2017), which our present work extends to the orthogonalized power envelope correlation technique. Consistent with our guideline, the MEG studies that identified the PMC as a core hub for dynamic brain integration did rely on a version of MNE (de Pasquale et al., 2012, 2016).

This simple message may have a profound impact on the field because of the critical role of the DMN in the human brain. This holds especially true for the PMC itself, which is singled out as a major cortical area for both structural and functional connectivity (Leech and Sharp, 2014) and as one core dynamic integrator of other RSNs (de Pasquale et al., 2012, 2016), and thus plays a pivotal role in healthy brain functioning. This multifaceted centrality of the PMC explains its prominent involvement in a wide spectrum of major neurological and psychiatric disorders (Leech and Sharp, 2014). Abnormalities in PMC activity and its interactions within the DMN have indeed been consistently reported in relation with traumatic brain injuries, epilepsy, multiple sclerosis, and neuropsychiatric disorders such as schizophrenia, autism, attention deficit hyperactivity disorders, or depression (Fox, 2010; Leech and Sharp, 2014). The reason is presumably that those PMC/DMN abnormalities are merely epiphenomena induced by connectional diaschisis (i.e., a loss or change of FC between brain areas connected to a distant, but lesioned, brain area), since brain hubs are more likely to be affected in any network-level disorder (Buckner, 2005). However, this consistent involvement of the PMC might bring novel insights into the pathophysiology of fundamentally distinct brain disorders and to novel diagnostic or prognostic procedures in the future. The PMC and the DMN are also directly impaired in neurodegenerative disorders such as Alzheimer's disease, which have been related to prominent amyloid and tau protein depositions in the PMC and the other areas of the DMN (Buckner, 2005; Park et al., 2014). Importantly, in this pathological situation both neurometabolism and neurovascularisation are affected (Hampel et al., 2011), hence making it more crucial to estimate DMN functional interactions by proper electrophysiological methods. Considering the increasingly aging population worldwide, Alzheimer's disease is bound to become the major health challenge of the $21^{\text {st }}$ century, with weighty social and economic impacts (Hampel et al., 2011). In this context, the ability to investigate non-invasively the electrophysiology of PMC connectivity within the DMN as well as its dynamic cross-network FC appears essential. Therefore, our study should prompt future studies along these lines of research to consider MNE rather than LCMV Beamformer for the reconstruction and analysis of DMN functional connectivity.

Still, a systematic usage of both reconstruction methods may also prove worthwhile to investigate possible modulations of within-DMN linear synchrony in brain disorders. Actually, to the best of our knowledge, our study is the first to provide scientific conclusions about neurophysiology based on a comparison of distinct source reconstruction methods and usage of their analytical properties. As such, it demonstrates the general potential of this approach to generate novel insights about human 
brain functional dynamics.

\section{Acknowledgements}

M.S. was supported by a research grant by the WienerAnspach Foundation (Brussels, Belgium and Oxford, UK) and is supported by the Research Convention "Marc Errens" of the Fonds Erasme (Brussels, Belgium). X.D.T. is Post-doctorate Clinical Master Specialist at the Fonds de la Recherche Scientifique (F.R.S.-FNRS, Brussels, Belgium). J.V.S. was supported by a FWO $(12 \mathrm{I} 1817 \mathrm{~N}$, Brussels, Belgium). This research project was supported by research grants from the WienerAnspach Foundation (Brussels, Belgium and Oxford, UK) and by the Fonds Erasme (Research Convention "Marc Errens", Brussels, Belgium). The MEG project at the CUB - Hôpital Erasme is financially supported by the Fonds Erasme (Research Convention "Les Voies du Savoir", Brussels, Belgium).

\section{Supplementary material}

\section{S1. Robustness of empirical results against precise seed posi- tioning}

To confirm that our results depicted in Fig. 1 are not specific to our particular choice of seed location within the $\mathrm{MPFC}$, we generated similar seed-based FC maps with the original seed moved $1 \mathrm{~cm}$ forward and backward along the three MNI coordinate axes. The six resulting sets of maps are depicted in Fig. S1. The topographies appeared essentially unaffected by seed translations, so that our qualitative results described in the main text stand.

\section{S2. Robustness of empirical results against precise choice of DMN regions of interest}

Similarly, we checked that the particular size and location of the ROI masks used in Fig. 2 were not determining factors for our statistical analyses of the reconstruction- and systemtype effects. We thus repeated our analysis by replacing the full-sized parcels by half-sized parcels (i.e., by modulating the threshold applied to the weighted ROI maps), or by translating the parcels by $1 \mathrm{~cm}$ both forward and backward along the MNI coordinate axes. We then repeated the two-way ANOVA on each of the eight resulting FC matrices. The significant main effect of reconstruction on the mPFC-PMC coupling estimate subsisted in all cases $\left(F_{1,56} \geq 33.4, p \leq 3.5 \times 10^{-7}\right.$, uncorrected), as well as the absence of significant system type or interaction effect on this coupling $\left(F_{1,56} \leq 1.1, p \geq .3\right)$. No other connection disclosed any significant effect $\left(F_{1,56} \leq 4.95\right.$, $p \geq .03)$. Therefore, our results reported in the main text are unaffected by the precise size and location of the ROIs used.

\section{S3. Impact of source-space grid resolution on the mPFC-PMC coupling estimate}

To exclude the possibility that the LCMV Beamformerspecific absence of FC between MPFC and PMC was not due to a source sampling bias, we used a $2-\mathrm{mm}$ regular grid to estimate the FC of the DMN. The resulting mPFC-based FC maps were essentially unaffected, even those obtained with the LCMV Beamformer (Fig. S2, top). Comparing the LCMV Beamformer-based mPFC-PMC coupling estimates with the coarse and fine grids (Fig. S2, bottom) did not reveal any difference in either of the system types $\left(t_{14}<.3, p>.75\right)$.

\section{S4. Impact of one-dimensional source projection on connectiv- ity estimation}

The FC pipeline used here relied on point source estimates projected onto their direction of maximum variance (as in, e.g., Brookes et al. 2011 and Wens et al., 2014), so as to estimate their Hilbert envelope and correlate them. However, this approach discards a substantial part of the source variance. In our data, the mean fraction of variance (across sources, subjects, and MEG systems) retained by the principal component was $67 \%$ for MNE and $70 \%$ for the LCMV Beamformer. Other pipelines consider the correlation between band-limited power estimated as the squared Euclidean norm of three-dimensional sources (as in, e.g., de Pasquale et al., 2010; 2012). However these two approaches lead to very similar results, at least for resting-state FC. For example, we re-estimated using the latter method the FC between mPFC and PMC, which is key to our results, and found remarkably similar values with our onedimensional source estimates in any of the four combination of reconstruction and system $\left(t_{14}<.5, p>.62\right)$.

\section{S5. Necessity of increased PMC variance in point-like PMC simulations}

In the point-like simulations of the DMN discussed in the main text, we boosted the variance of the PMC signal by a subject-specific factor related to its cortical depth relative to that of the TPJs. The rationale for this is that activity from deep sources contributes less to the sensor signals due to their larger distance to the sensors array. We illustrate the necessity of this PMC boost by repeating the simulation shown in Fig. 3A, except that the PMC was either absent (Fig. S3, left) or of variance equal to that of the TPJs (Fig. S3, middle). In both cases, the PMC was completely absent from the seed-based FC maps. Boosting the PMC signal is thus necessary to make it contribute (Fig. S3, right). Because the simulation considered here did not include any linear, "zero-lag" correlation, this observation holds equally well for both MNE (Fig. S3, top) and the LCMV Beamformer (Fig. S3, bottom).

\section{S6. Establishing network-independent FC reduction with the LCMV Beamformer}

To illustrate the observation that the global FC reduction due to the LCMV Beamformer is independent of brain network activity, we considered a pure noise simulation wherein no DMN node was active. The results are illustrated in Fig. S4. Topographical maps for both MNE and LCMV Beamformer appeared random, as expected. However, the average FC value was twice as large when using MNE than LCMV Beamformer. 


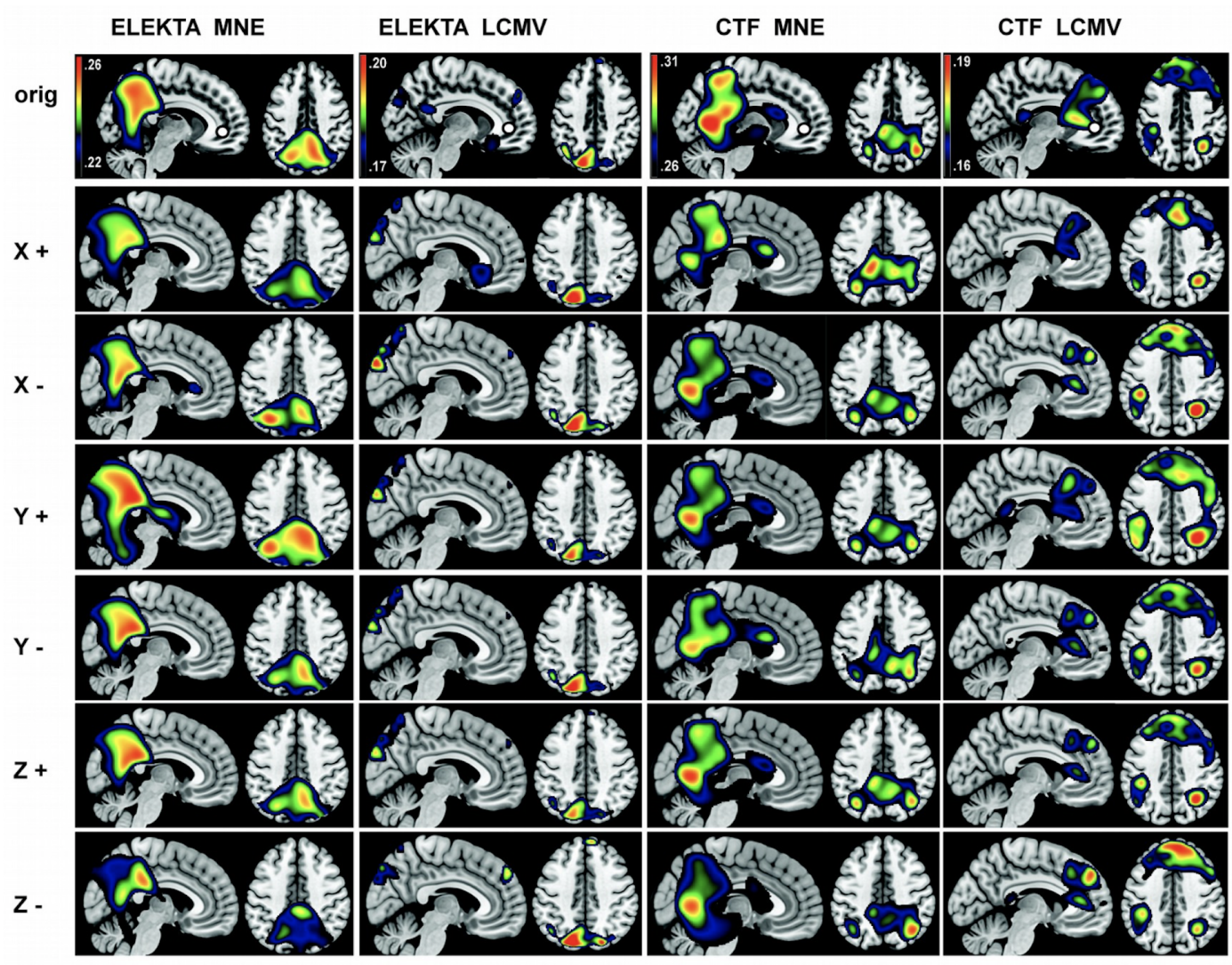

Figure S1: Stability of seed-based DMN topography under seed translation. The mPFC-based FC maps derived with two reconstruction algorithms (MNE and LCMV Beamformer) and two MEG system types (Elekta and CTF) are shown for the original seed location (top row, see also Fig. 1) and for an mPFC seed translated $1 \mathrm{~cm}$ forward (+) and backward (-) along the $X, Y$, and $Z$ axes of the MNI coordinate frame. For example, the row denoted $X+$ corresponds to the original seed translated by $1 \mathrm{~cm}$ to the right. 

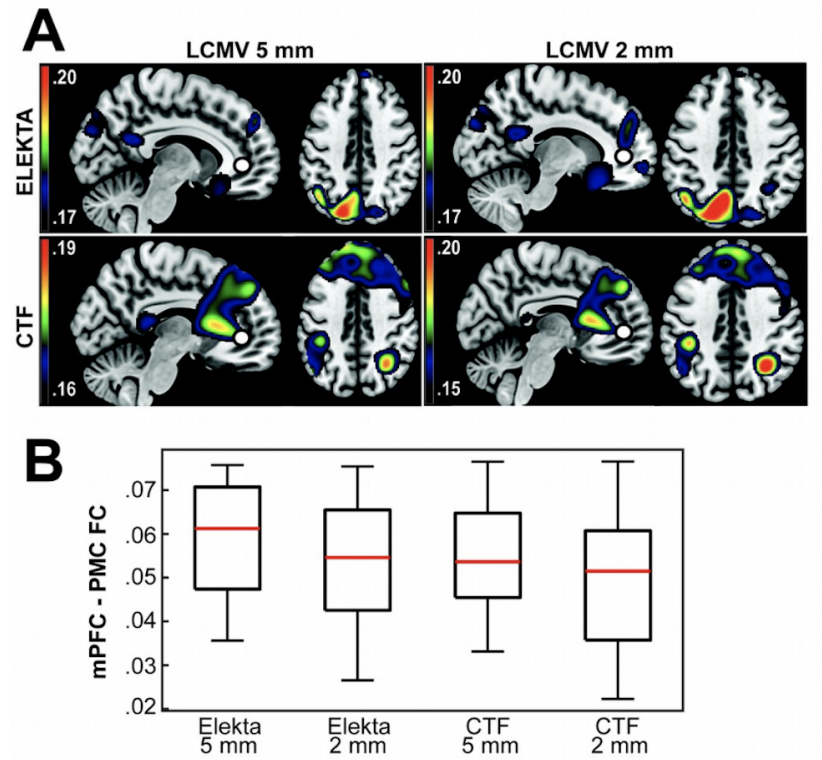

Figure S2: Effect of precise resolution of source space grid on LCMV Beamformer-based connectivity. A: mPFC-based envelope FC topographies for both MEG system (Elekta and CTF) and grid spacing (2 mm vs $5 \mathrm{~mm}$ ). B: Boxplots showing the corresponding mPFC-PMC coupling estimates.

\section{S7. Necessity of bi-laterality for the linear PMC-TPJ coupling in point-like PMC simulations}

We argued in the main text that the point-like PMC scenario required a bilateral PMC-TPJ correlation in order to reproduce the LCMV Beamformer-specific reduction of FC with the PMC, while preserving FC with both left and right TPJs. To illustrate this, we consider here a similar simulation based on a unilateral correlation between the PMC and the left TPJ. Specifically, the PMC and left TPJ signals were identical and linearly independent from the right TPJ signal. The resulting seed-based FC map clearly shows a LCMV Beamformer-specific disappearance of both the PMC and the left TPJ, and preservation of the right TPJ (Fig. S5).

\section{References}

[1] Allen, E.A., Damaraju, E., Plis, S.M., Erhardt, E.B., Eichele, T., Calhoun, V.D., 2014. Tracking whole-brain connectivity dynamics in the resting state. Cerebr. Cortex 24, 663-676. https://doi.org/10.1093/cercor/bhs352.

[2] Ashburner, J., Friston, K.J., 1999. Nonlinear spatial normalization using basis functions. Hum. Brain Mapp. 7, 254-266. https://doi.org/10.1002/(SICI)1097-0193(1999)7: 4\%3c254i::AIDHBM4\%3e3.0.CO;2-G.

[3] Baars, B.J., Ramsy, T.Z., Laureys, S., 2003. Brain, conscious experience and the observing self. Trends Neurosci. 26, 671-675. https://doi.org/10.1016/j.tins.2003.0 9.015.

[4] Boly, M., Balteau, E., Schnakers, C., Degueldre, C., Moonen, G., Luxen, A., Phillips, C., Peigneux, P., Maquet, P., Laureys, S., 2007. Baseline brain activity fluctuations predict somatosensory perception in humans. Proc. Natl. Acad. Sci. Unit. States Am. 104, 12187-12192. https://doi.org/10.1073/pnas.0611404104.

[5] Brookes, M.J., Woolrich, M., Luckhoo, H., Price, D., Hale, J.R., Stephenson, M.C., Barnes, G.R., Smith, S.M., Morris, P.G., 2011. Investigating the electrophysiological basis of resting state networks using magnetoencephalography. Proc. Natl. Acad. Sci. Unit. States Am. 108, 16783-16788. https://doi.org/10.1073/pnas.1112685108.
[6] Brookes, M.J., Woolrich, M.W., Barnes, G.R., 2012. Measuring functional connectivity in MEG: a multivariate approach insensitive to linear source leakage. Neuroimage 63, 910-920. https://doi.org/10.1016/j.neuroimage.2012.03.048.

[7] Buckner, R.L., 2005. Molecular, structural, and functional characterization of Alzheimer's disease: evidence for a relationship between default activity, amyloid, and memory. J. Neurosci. 25, 7709-7717. https://doi.org/10.1523/JNEUROSCI.2177-05.2005.

[8] Buckner, R.L., Andrews-Hanna, J.R., Schacter, D.L., 2008. The brain's default network: anatomy, function, and relevance to disease. Ann. N. Y. Acad. Sci. 1124, 1-38. https ://doi.org/10.1196/annals.1440.011.

[9] Buzsaki, G., Anastassiou, C.A., Koch, C., 2012. The origin of extracellular fields and currents - EEG, ECoG, LFP and spikes. Nat. Rev. Neurosci. 13, 407-420. https://doi. org/10.1038/nrn3241.

[10] Cavanna, A.E., Trimble, M.R., 2006. The precuneus: a review of its functional anatomy and behavioural correlates. Brain 129, 564-583. https://doi.org/10.1093/brain/a w1004.

[11] Colclough, G.L., Brookes, M.J., Smith, S.M., Woolrich, M.W., 2015. A symmetric multivariate leakage correction for MEG connectomes. Neuroimage 117, 439-448. https://doi.org/10.1016/j.neuroimage.2015.03.071.

[12] Coquelet, N., Mary, A., Peigneux, P., Goldman, S., Wens, V., De Tiège, X., 2017. The electrophysiological connectome is maintained in healthy elders: a power envelope correlation MEG study. Sci. Rep. 7. https://doi.org/10.1038/s41598-017-13829-8.

[13] Dale, A., Sereno, M., 1993. Improved localization of cortical activity by combining EEG and MEG with MRI cortical surface reconstruction. J. Cogn. Neurosci. 20.

[14] de Pasquale, F., Della Penna, S., Snyder, A.Z., Lewis, C., Mantini, D., Marzetti, L., Belardinelli, P., Ciancetta, L., Pizzella, V., Romani, G.L., Corbetta, M., 2010. Temporal dynamics of spontaneous MEG activity in brain networks. Proc. Natl. Acad. Sci. Unit. States Am. 107, 6040-6045. https://doi.org/10.1073/pnas.0913863107.

[15] de Pasquale, F., DellaPenna, S., Snyder, A.Z., Marzetti, L., Pizzella, V., Romani, G.L., Corbetta, M., 2012. A cortical core for dynamic integration of functional networks in the resting human brain. Neuron 74, 753-764. https://doi.org/10.1016/j.neuron.20 12.03.031.

[16] de Pasquale, F., Della Penna, S., Sporns, O., Romani, G.L., Corbetta, M., 2016. A dynamic core network and global efficiency in the resting human brain. Cerebr. Cortex 26, 4015-4033. https://doi.org/10.1093/cercor/bhv185.

[17] de Pasquale, F., Corbetta, M., Betti, V., Della Penna, S., 2018. Cortical cores in network dynamics. Neuroimage 180, 370-382. https://doi.org/10.1016/ j.neuroimage.2017.09.063.

[18] Deco, G., Corbetta, M., 2011. The dynamical balance of the brain at rest. Neuroscientist 17, 107-123. https://doi.org/10.1177/1073858409354384.

[19] D'Esposito, M., Deouell, L.Y., Gazzaley, A., 2003. Alterations in the BOLD fMRI signal with ageing and disease: a challenge for neuroimaging. Nat. Rev. Neurosci. 4, 863-872. htt ps://doi.org/10.1038/nrn1246.

[20] Fischl, B., 2012. FreeSurfer. NeuroImage 62, 774-781. https://doi.org/10.1016/j.neu roimage.2012.01.021.

[21] Fox, M.D., 2010. Clinical applications of resting state functional connectivity. Front. Syst. Neurosci. https://doi.org/10.3389/fnsys.2010.00019.

[22] Fox, M.D., Raichle, M.E., 2007. Spontaneous fluctuations in brain activity observed with functional magnetic resonance imaging. Nat. Rev. Neurosci. 8, 700-711. https://doi. org/10.1038/nrn2201.

[23] Friston, K., Ashburner, J., Kiebel, S., Nichols, T., Penny, W., 2007. Statistical Parametric Mapping: the Analysis of Functional Brain Images. Academic Press.

[24] Gramfort, A., Luessi, M., Larson, E., Engemann, D.A., Strohmeier, D., Brodbeck, C., Parkkonen, L., Hamalainen, M.S., 2014. MNE software for processing MEG and EEG data. Neuroimage 86, 446-460. https://doi.org/10.1016/j.neuroimage.2013.10.027.

[25] Greicius, M.D., Krasnow, B., Reiss, A.L., Menon, V., 2003. Functional connectivity in the resting brain: a network analysis of the default mode hypothesis. Proc. Natl. Acad. Sci. Unit. States Am. 100, 253-258. https://doi.org/10.1073/pnas.0135058100.

[26] Hagmann, P., Cammoun, L., Gigandet, X., Meuli, R., Honey, C.J., Wedeen, V.J., Sporns, O., 2008. Mapping the structural core of human cerebral cortex. PLoS Biol. 6, e159. https://doi.org/10.1371/journal.pbio.0060159.

[27] Hampel, H., Prvulovic, D., Teipel, S., Jessen, F., Luckhaus, C., Frolich, 

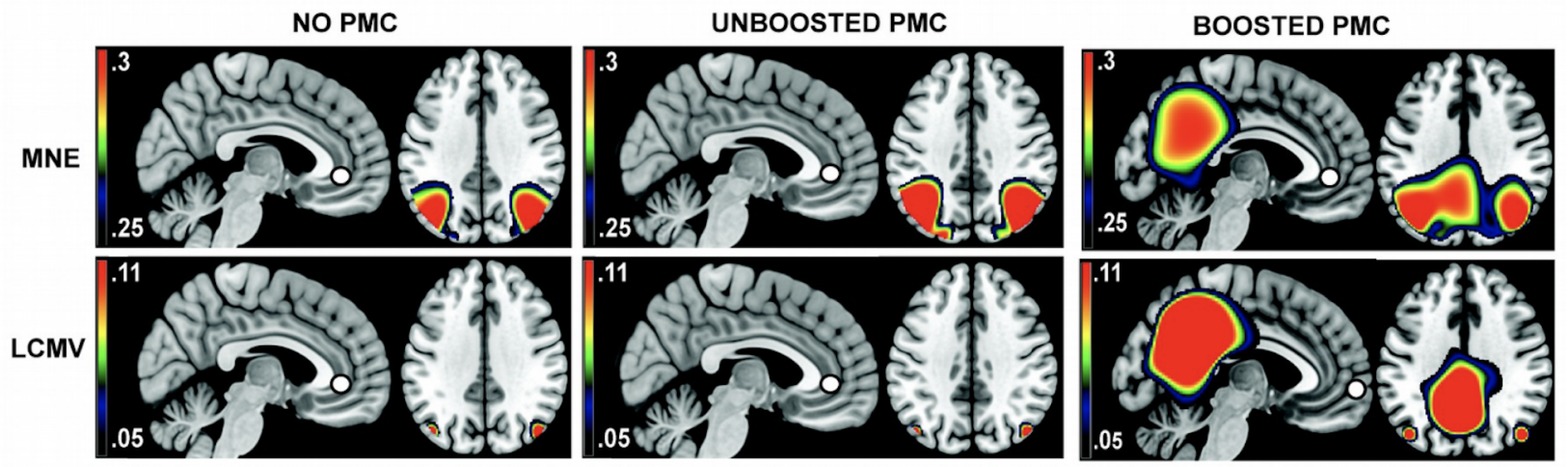

Figure S3: Necessity of increased PMC variance in a point-like PMC simulation. The mPFC-based FC maps built with two reconstruction algorithms (MNE and LCMV Beamformer) were generated using a point-like PMC simulation of the DMN without PMC-TPJ coupling but with varying PMC variance: zero variance, i.e., no PMC was included (left), variance equal to that of the TPJs, i.e., without accounting for its depth-related loss of signal (middle), and with boosted variance according to its cortical depth relative to the TPJs (right, see also top row of Fig. 3).

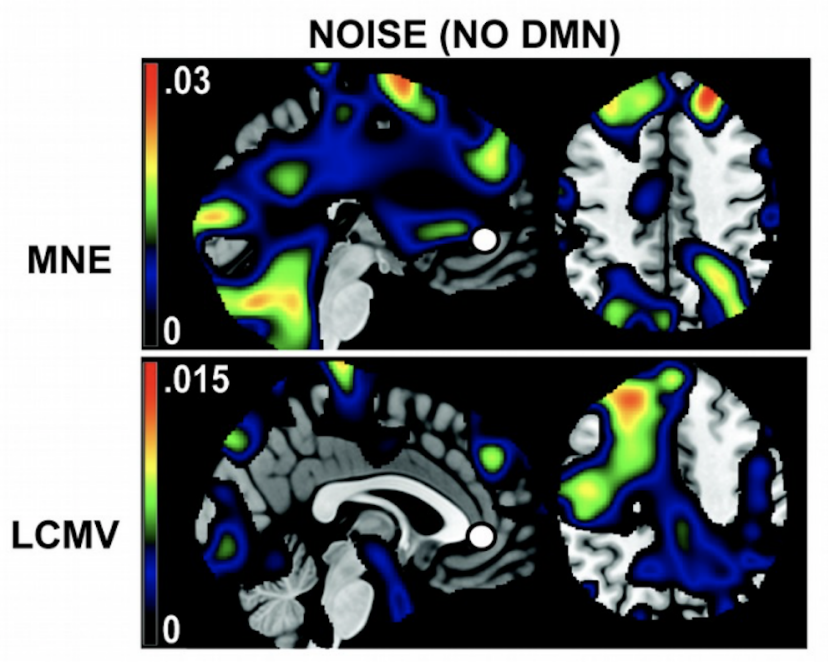

Figure S4: Establishing network-independent reduction of FC with the LCMV Beamformer. The mPFC-based FC maps built with two reconstruction algorithms (MNE and LCMV Beamformer) were generated using a pure noise simulation with no network activity.

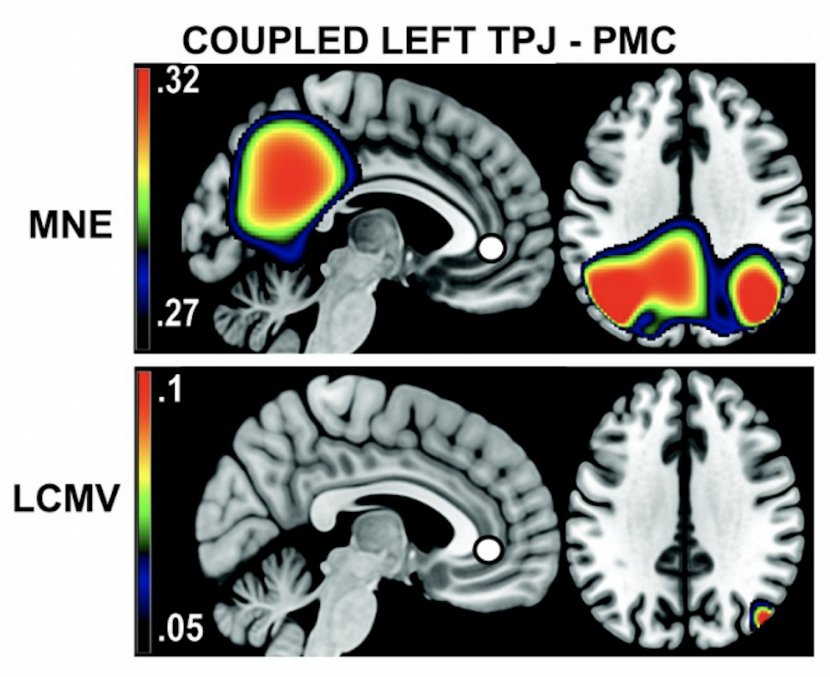

Figure S5: Necessity of bi-laterality of the linear PMC-TPJ coupling in pointlike PMC simulations. The mPFC-based FC maps built with two reconstruction algorithms (MNE and LCMV Beamformer) were generated using a point-like PMC simulation that included a linear, "zero-lag" correlation between the PMC and the left TPJ. 
L., Riepe, M.W., Dodel, R., Leyhe, T., Bertram, L., Hoffmann, W., Faltraco, F., 2011. The future of Alzheimer's disease: the next 10 years. Prog. Neurobiol. 95, 718-728. https:// doi.org/10.1016/j.pneurobio.2011.11.008.

[28] Hillebrand, A., Singh, K.D., Holliday, I.E., Furlong, P.L., Barnes, G.R., 2005. A new approach to neuroimaging with magnetoencephalography. Hum. Brain Mapp. 25, 199-211. https://doi.org/10.1002/hbm.20102.

[29] Hincapié, A.-S., Kujala, J., Mattout, J., Pascarella, A., Daligault, S., Delpuech, C., Mery, D., Cosmelli, D., Jerbi, K., 2017. The impact of MEG source reconstruction method on source-space connectivity estimation: a comparison between minimum-norm solution and beamforming. Neuroimage 156, 29-42. https://doi.org/10.1016/j.neuroimage. 2017.04.038.

[30] Hipp, J.F., Hawellek, D.J., Corbetta, M., Siegel, M., Engel, A.K., 2012. Large-scale cortical correlation structure of spontaneous oscillatory activity. Nat. Neurosci. 15. htt ps://doi.org/10.1038/nn.3101.

[31] Hyvarinen, A., Oja, E., 2000. Independent component analysis: algorithms and applications. Neural Netw 13, 411-430. https://doi.org/10.1016/S0893-6080(00) 00026-5

[32] Jerbi, K., Vidal, J.R., Ossandon, T., Dalal, S.S., Jung, J., Hoffmann, D., Minotti, L., Bertrand, O., Kahane, P., Lachaux, J.P., 2010. Exploring the electrophysiological correlates of the defaultmode network with intracerebral EEG. Front. Syst. Neurosci. 4. https://doi.org/10.3389/fnsys.2010.00027.

[33] Leech, R., Sharp, D.J., 2014. The role of the posterior cingulate cortex in cognition and disease. Brain 137, 12-32. https://doi.org/10.1093/brain/awt162.

[34] Liu, Q., Farahibozorg, S., Porcaro, C., Wenderoth, N., Mantini, D., 2017. Detecting large-scale networks in the human brain using high-density electroencephalography: imaging Brain Networks with High Density EEG. Hum. Brain Mapp. 38, 4631-4643. https://doi.org/10.1002/hbm.23688.

[35] Liu, T.T., 2013. Neurovascular factors in restingstate functional MRI. Neuroimage 80, 339-348. https://doi.org/10.1016/j.neuroimage.2013.04.071.

[36] Margulies, D.S., Vincent, J.L., Kelly, C., Lohmann, G., Uddin, L.Q., Biswal, B.B., Villringer, A., Castellanos, F.X., Milham, M.P., Petrides, M., 2009. Precuneus shares intrinsic functional architecture in humans and monkeys. Proc. Natl. Acad. Sci. Unit. States Am. 106, 20069-20074. https://doi.org/10.1073/pnas.0905314106.

[37] Marzetti, L., Di Lanzo, C., Zappasodi, F., Chella, F., Raffone, A., Pizzella, P., 2014. Magnetoencephalographic alpha band connectivity reveals differential default mode network interactions during focused attention and open monitoring meditation. Front. Hum. Neurosci. 8. https://doi.org/10.3389/fnhum.2014.00832.

[38] Niso, G., Rogers, C., Moreau, J.T., Chen, L.-Y., Madjar, C., Das, S., Bock, E., Tadel, F., Evans, A.C., Jolicoeur, P., Baillet, S., 2016. OMEGA: the open MEG archive. Neuroimage 124, 1182-1187. https://doi.org/10.1016/j.neuroimage.2015.04.028. O'Neill, G.C., Barratt, E.L., Hunt, B.A.E., Tewarie, P.K., Brookes, M.J., 2015. Measuring electrophysiological connectivity by power envelope correlation: a technical review on MEG methods. Phys. Med. Biol. 60, R271-R295. https://doi.org/10.1088/00 31-9155/60/21/R271.

[39] Palva, J.M., Wang, S.H., Palva, S., Zhigalov, A., Monto, S., Brookes, M.J., Schoffelen, J.M., Jerbi, K., 2018. Ghost interactions in MEG/EEG source space: a note of caution on inter-areal coupling measures. Neuroimage 173, 632-643. https://doi.org/10.10 16/j.neuroimage.2018.02.032.

[40] Park, L., Koizumi, K., El Jamal, S., Zhou, P., Previti, M.L., Van Nostrand, W.E., Carlson, G., Iadecola, C., 2014. Age-dependent neurovascular dysfunction and damage in a mouse model of cerebral amyloid angiopathy. Stroke 45, 1815-1821. https://doi.org/10.11 61/STROKEAHA.114.005179.

[41] Raichle, M.E., 2015. The brain's default mode network. Annu. Rev. Neurosci. 38, 433-447. https://doi.org/10.1146/annurev-neuro-071013-014030.

[42] Raichle, M.E., MacLeod, A.M., Snyder, A.Z., Powers, W.J., Gusnard, D.A., Shulman, G.L., 2001. A default mode of brain function. Proc. Natl. Acad. Sci. Unit. States Am. 98, 676-682. https://doi.org/10.1073/pnas.98.2.676.

[43] Savio, A., Fnger, S., Tahmasian, M., Rachakonda, S., Manoliu, A., Sorg, C., Grimmer, T., Calhoun, V., Drzezga, A., Riedl, V., Yakushev, I., 2017. Resting-state networks as simultaneously measured with functional MRI and PET. J. Nucl. Med. 58, 1314-1317. https://doi.org/10.2967/jnumed.116.185835.

[44] Siegel, M., Donner, T.H., Engel, A.K., 2012. Spectral fingerprints of large-scale neuronal interactions. Nat. Rev. Neurosci. 13, 121-134. https://doi.org/10.1038/nrn3137. Siems, M., Pape, A.-A., Hipp, J.F., Siegel, M., 2016. Measuring the cortical correlation structure of spontaneous oscillatory activity with EEG and MEG. Neuroimage 129, 345-355. https://doi.org/10.1016/j.neuroimage.2016.01.055.

[45] Smith, S.M., Fox, P.T., Miller, K.L., Glahn, D.C., Fox, P.M., Mackay, C.E., Filippini, N., Watkins, K.E., Toro, R., Laird, A.R., Beckmann, C.F., 2009. Correspondence of the brain's functional architecture during activation and rest. Proc. Natl. Acad. Sci. Unit. States Am. 106, 13040-13045. https://doi.org/10.1073/pnas.0905267106

[46] Sporns, O., Honey, C.J., Kotter, R., 2007. Identification and classification of hubs in brain networks. PLoS One 2, e1049. https://doi.org/10.1371/journal.pone.0001049.

[47] Taulu, S., Simola, J., Kajola, M., 2005. Applications of the signal space separation method. IEEE Trans. Signal Process. 53, 3359-3372. https://doi.org/10.1109/TSP.200 5.853302.

[48] Trotta, N., Baete, K., Laere, K.V., Goldman, S., Ti ege, X.D., Wens, V., 2018. Neurometabolic resting-state networks derived from seed-based functional connectivity analysis. J. Nucl. Med. Off. Publ. Soc. Nucl. Med. 59, 1642-1643. htt ps://doi.org/10.2967/jnumed.118.212878.

[49] Utevsky, A.V., Smith, D.V., Huettel, S.A., 2014. Precuneus is a functional core of the default-mode network. J. Neurosci. 34, 932-940. https://doi.org/10.1523/JNE UROSCI.4227-13.2014.

[50] Van Veen, B.D., 1997. Localization of brain electrical activity via linearly constrained minimum variance spatial filtering. IEEE Trans. Biomed. Eng. 44, 14.

[51] Vidaurre, D., Hunt, L.T., Quinn, A.J., Hunt, B.A.E., Brookes, M.J., Nobre, A.C., Woolrich, M.W., 2018. Spontaneous cortical activity transiently organises into frequency specific phase-coupling networks. Nat. Commun. 9. https://doi.org/10.1 038/s41467-018-05316-z.

[52] Vigario, R., Sarela, J., Jousmaki, V., Hamalainen, M., Oja, E., 2000. Independent component approach to the analysis of EEG and MEG recordings. IEEE Trans. Biomed. Eng. 47, 5.

[53] Vogt, B.A., Vogt, L., Laureys, S., 2006. Cytology and functionally correlated circuits of human posterior cingulate areas. Neuroimage 29, 452-466. https://doi.org/10.10 16/j.neuroimage.2005.07.048.

[54] Wens, V., Marty, B., Mary, A., Bourguignon, M., Op de Beeck, M., Goldman, S., Van Bogaert, P., Peigneux, P., De Tiège, X., 2015. A geometric correction scheme for spatial leakage effects in MEG/EEG seed-based functional connectivity mapping: spatial Leakage Geometric Correction Scheme. Hum. Brain Mapp. 36, 4604-4621. https://doi.org/10.1002/hbm.22943.

[55] Wens, V., Mary, A., Bourguignon, M., Goldman, S., Marty, B., Op de Beeck, M., Bogaert, P.V., Peigneux, P., De Tiège, X., 2014. About the electrophysiological basis of resting state networks. Clin. Neurophysiol. 125, 1711-1713. https://doi.org/10.10 16/j.clinph.2013.11.039.

[56] Xu, J., Vik, A., Groote, I.R., Lagpopoulos, J., Holen, A., Ellingsen, ., Håberg, A.K., Davanger, S., 2014. Nondirective meditation activates default mode network and areas associated with memory retrieval and emotional processing. Front. Hum. Neurosci. 8. https://doi.org/10.3389/fnhum.2014.00086. 